Check for updates

Cite this: RSC Adv., 2017, 7, 50317

Received 30th August 2017

Accepted 20th October 2017

DOI: $10.1039 / c 7 r a 09622 d$

rsc.li/rsc-advances

\section{Different toxicity of cadmium telluride, silicon, and carbon nanomaterials against hemocytes in silkworm, Bombyx mori†}

\author{
Kai-Le Li, $\ddagger^{\text {ab }}$ Yun-Hu Zhang, $t^{\text {ab }}$ Rui Xing, ${ }^{\text {ab }}$ Yan-Feng Zhou, ${ }^{c}$ Xue-Dong Chen, ${ }^{\text {ab }}$ \\ Hao Wang, ${ }^{d}$ Bin Song, ${ }^{c}$ Yang-Hu Sima, ${ }^{\text {b }}$ Yao He (iD ${ }^{* c}$ and Shi-Qing Xu (D) *ab
}

The biological toxicity of nanomaterials is a concern because they have many potential applications in biomedical fields. In this study, silkworm hemolymph was exposed to high-dose cadmium telluride quantum dots (CdTe QDs), citric-acid-nitrogen-doped carbon dots (C-NCDs), or silicon nanoparticles (SiNPs) and the differences in the immune responses and programmed cell death induced in hemocytes were compared. Changes in the expression of innate-immunity-related genes and the occurrence of hemocytes in the hemolymph indicated that the three types of nanomaterials entered several types of hemocytes by endocytosis, but their toxicity differed significantly. C-NCDs only induced autophagy in the hemocytes, whereas SiNPs induced both hemocyte autophagy and the simultaneous apoptosis of a large number of cells. CdTe QD exposure rapidly induced hemocyte apoptosis and necrosis. The mechanisms of hemocyte apoptosis induced by the different nanomaterials differed significantly. The apoptosis induced by CdTe QDs was dependent on the lysosomal apoptotic pathway, whereas SiNPs also used the endoplasmic reticulum apoptotic pathway. The autophagy and even apoptosis that appeared in the hemocytes after SiNP exposure quickly self-repaired, whereas the autophagy induced in hemocytes by $\mathrm{C}-\mathrm{NCD}$ exposure persisted.

\section{Introduction}

Nanomaterials of quantum dots (QDs), silicon- or carbon-based nanoparticles, etc. have potentially broad applications in biomedical fields, including in biological imaging, ${ }^{1-3}$ targeted drug delivery, and disease treatment. ${ }^{4-7}$ They are also beginning to be used in products such as cosmetics, food additives, and sporting equipment. ${ }^{8}$ Therefore, their biosafety has received much attention. Intravenous or subcutaneous injection, taking orally, inhalation and skin touch are the main clinical routes of nanomaterials administration, so an in-depth investigation of the effects of nanomaterials in the blood circulation and on

${ }^{a}$ School of Biology and Basic Medical Sciences, Medical College, Soochow University, Suzhou 215123, China. E-mail: szsqxu@suda.edu.cn

${ }^{b}$ National Engineering Laboratory for Modern Silk (NESER), Soochow University, Suzhou 215123, China

'Jiangsu Key Laboratory for Carbon-Based Functional Materials and Devices, Institute of Functional Nano and Soft Materials (FUNSOM), Collaborative Innovation Center of Suzhou Nano Science and Technology (NANO-CIC), Soochow University, Suzhou 215123, China. E-mail: yaohe@suda.edu.cn

${ }^{a}$ Soochow Institute for Energy and Materials Innovations, College of Physics, Optoelectronics and Energy, Collaborative Innovation Center of Suzhou Nano Science and Technology, Soochow University, Suzhou 215006, China

$\dagger$ Electronic supplementary information (ESI) available. See DOI: $10.1039 / \mathrm{c} 7 \mathrm{ra09622d}$

$\ddagger$ These authors contributed equally to this work. hematopoietic functions is required. Thus far, assessment of the toxicity of nanomaterials in animals, such as mice, through tracheal instillation or inhalation has been reported., ${ }^{\mathbf{9} 10}$ After the intravenous injection of nanomaterials in mice, they reach multiple organs in the body within $30 \mathrm{~min}$, and accumulate at an effective concentration in the liver and bladder. ${ }^{\mathbf{1 1 - 1 3}}$ Recent studies have focused on the in vivo transport kinetics of nanomaterials, ${ }^{14}$ the phagocytosis of nanomaterials by hemocytes, ${ }^{15}$ and the changes in the immune response they induce in the blood. ${ }^{16}$ However, the mechanisms underlying the differences in toxicity of nanomaterials against circulating hemocytes and the activation of the innate immune responses have not yet been reported.

Several issues have been highlighted in the selection of experimental animals for nano-toxicology. The use of classic mammalian model animals such as mice and monkeys in toxicological research into rapidly developing nanomaterials not only entails high costs and long experimental periods, but involves bioethical issues. ${ }^{17}$ Moreover, the cytokine networks of the complex mammalian immune system ${ }^{\mathbf{1 8}}$ means that the results of these studies often do not fully assess the hematoxicity of nanomaterials and their effects on hematopoietic functions. Therefore, other representative tissues and systematic models are required to evaluate the safety of nanomaterials. ${ }^{19-21}$ Invertebrate hemocytes are widely considered to be a superior model with which to study innate immunity and 
DNA damage..$^{20-23}$ The innate immune system of the silkworm, an invertebrate model organism, has many characteristics in common with the vertebrate immune system, but without the interference of the complex acquired immune system of mammals. ${ }^{\mathbf{2 4 2 5}}$ Another advantage is that quantum dots are distributed evenly throughout the bodies of silkworm larvae within 1 min of their injection via the dorsal vein. ${ }^{20}$ All the visceral tissues of the silkworm, including the hematopoietic organs (HOs), are infiltrated with hemolymph, so the experimental results can be used to compare the differences between in vitro cell culture and in vivo experiments. ${ }^{26}$ This circumvents the effects of the tissue barriers of mammals and eliminates the errors caused by the inhibition of angiogenesis by the tested drugs. ${ }^{27}$ Silkworms also repeat the stable formation and release of hemocytes from the HOs in vitro, so they can be used to investigate hematopoietic toxicology both in vivo and in vitro, which is difficult to achieve in mammals. ${ }^{20,28}$ Therefore, the use of silkworms to assess the toxic effects of nanomaterials on hemocytes and hematopoietic functions has unique advantages.

In this study, the circulating hemocytes and HOs of silkworm larvae were selected to evaluate the mechanisms underlying the differences in toxicity on circulating hemocytes of three representative nanomaterials.

\section{Experimental}

\section{Preparation of nanomaterials}

Cadmium telluride quantum dots (CdTe QDs) and silicon nanoparticles (SiNPs) were synthesized through our previously reported microwave-assisted synthetic methods. ${ }^{29,30}$ Citric-acidnitrogen-doped carbon dots (C-NCDs) were synthesized by Professor Zou's team at the College of Physics, Optoelectronics, and Energy at Soochow University. Their characteristics have been reported by Zhong et al. ${ }^{29}$ Liu et al., ${ }^{20}$ and Wang et al. ${ }^{31}$ respectively. The corresponding hydrodynamic diameters of the CdTe QDs, SiNPs, and C-NCDs were 4, 2.2, and $11.4 \mathrm{~nm}$, respectively, and their maximum luminescent wavelengths were 530,450 , and $514 \mathrm{~nm}$, respectively.

\section{Preparation of experimental animals}

The Dazao strain of the silkworm, Bombyx mori, was provided by the National Engineering Laboratory for Modern Silk, Soochow University. The larvae were fed ad libitum with mulberry leaves, and kept in polypropylene cages $\left(30 \times 20 \times 12 \mathrm{~cm}^{3}\right)$ under a $12 \mathrm{~h}$ light/dark cycle, at a temperature of $25 \pm 2{ }^{\circ} \mathrm{C}$ and $70-$ $85 \%$ relative humidity. The research was performed according to the Soochow University Guidelines for the Welfare of Animals.

The sex-selection of newly exuviated fifth-instar larvae was based on the location and number of reproductive buds on the abdomen of the larvae, according to the method of Yan et al. ${ }^{32}$ We used 48 h-old fifth-instar larvae of similar bodyweight and the same sex. The nanomaterials $(10 \mu \mathrm{L})$ were injected via the dorsal vein after the larvae had been starved for $2 \mathrm{~h}$. Sterile ultra-pure water $(10 \mu \mathrm{L})$ was injected as the control. Our previous studies have shown that these nanomaterials exert significantly different toxic effects in silkworms, and that the optimal effective exposure causing hematoxicity is close to their maximum dissolved concentrations in water, these are $1 \mu \mathrm{g} \mu \mathrm{L}^{-1}$ for C-NCDs, $0.39 \mu \mathrm{g} \mu \mathrm{L}^{-1}$ for SiNPs, ${ }^{33}$ and $32 \mu \mathrm{M}$ for CdTe QDs. ${ }^{20}$

\section{Hemocyte observation and counting}

At $24 \mathrm{~h}$ after exposure to the nanomaterials, the larvae were pierced in the abdominal legs and $200 \mu \mathrm{L}$ of hemolymph was collected from each larva in an ice bath. The hemolymph $(600 \mu \mathrm{L})$ from three larvae was mixed and $10 \mu \mathrm{L}$ of a supersaturated solution of phenyl thiourea was rapidly added to the hemolymph sample. The hemolymph sample $(10 \mu \mathrm{L})$ was mounted and the characteristic fluorescence of the nanomaterials that entered the hemocytes was observed with a confocal laser scanning microscopy (CLSM; Leica TCS SP5, Wetzlar, Germany). Another $10 \mu \mathrm{L}$ of each sample was taken to determine the different types of hemocytes on a hemocyte counting plate (five replicates of each sample).

\section{Hemocyte staining}

The hemocytes were stained with propidium iodide (PI) (Cat. no. 287075; Sigma-Aldrich, Shanghai, China), terminal deoxynucleotidyl transferase (TdT)-mediated dUTP nick end labeling (TUNEL) (Cat. no. 12156792910; Sigma-Aldrich, Shanghai, China), and monodansylcadaverine (MDC) (Cat. no. 30432, Sigma-Aldrich, China). The hemocyte morphology and characteristic fluorescence after staining were observed with an Olympus SZX16 fluorescence microscope (Tokyo, Japan).

For PI staining, $10 \mu \mathrm{L}$ of the hemolymph sample was taken and an equal volume of $0.1 \mathrm{mg} \mathrm{mL}^{-1}$ PI working solution was added. The mixture was placed in an ice bath in the dark for $3 \mathrm{~min}$. An aliquot $(10 \mu \mathrm{L})$ of the mixture was removed to observe the characteristic fluorescence of PI, and the different types of hemocytes were counted according to their cell morphology (five replicates of each sample).

For TUNEL staining, $20 \mu \mathrm{L}$ of the hemolymph sample was mixed with $180 \mu \mathrm{L}$ of Hank's balanced salt solution (HBSS) on a slide. After the sample was allowed to stand for $5 \mathrm{~min}, 1 \mathrm{~mL}$ of cell fixative solution was added. After $15 \mathrm{~min}$, the sample was washed with phosphate-buffered saline (PBS). The hemolymph was incubated with cell permeabilization solution for $2 \mathrm{~min}$, and then washed three times with PBS for $5 \mathrm{~min}$ each. TUNEL reaction solution $(50 \mu \mathrm{L})$ was added dropwise and the sample was incubated at $37{ }^{\circ} \mathrm{C}$ for $60 \mathrm{~min}$ in a humidified chamber in the dark. After the sample was washed again with PBS, Antifade Mounting Medium (Cat. no. P0126, Beyotime, Jiangsu, China) was added dropwise. The TUNEL fluorescence was observed and photographed at wavelengths of 515-565 $\mathrm{nm}$.

For MDC staining, $10 \mu \mathrm{L}$ of each hemolymph sample was mixed with $10 \mu \mathrm{L}$ of MDC working solution and incubated at $37{ }^{\circ} \mathrm{C}$ in a water bath for $30 \mathrm{~min}$. An aliquot $(10 \mu \mathrm{L})$ of the mixture was placed on a slide for mounting. Fluorescence was observed at a wavelength of $512 \mathrm{~nm}$. 
The cytoplasmic calcium in the hemocytes was assayed with the membrane-permeable calcium indicator Xuo-3acetoxymethyl ester (Cat. no. S1056, Beyotime) and the lysosomes were stained with the Lyso-Tracker Red kit (Cat. no. C1046, Beyotime). For the calcium ion assay, $20 \mu \mathrm{L}$ of hemolymph sample was mixed with $180 \mu \mathrm{L}$ of HBSS. The samples were allowed to stand, the cells were fixed, and the slides were washed with PBS as described above for the TUNEL assay, and then $500 \mu \mathrm{L}$ of $5 \mu \mathrm{M}$ Fluo-3 AM working solution was added. The samples were reacted in the dark for $30 \mathrm{~min}$ and then washed three times with PBS for 5 min each. After Antifade Mounting Medium was added, the sample fluorescence was observed at a wavelength of $526 \mathrm{~nm}$. For lysosomal staining, $20 \mu \mathrm{L}$ of hemolymph sample was mixed with $10 \mu \mathrm{L}$ of $100 \mathrm{nM}$ lysosomal staining solution. After the sample was allowed to stand for $30 \mathrm{~min}$, the fluorescence was observed at a wavelength of $590 \mathrm{~nm}$.

\section{Immunofluorescence staining}

An primary antibody of caspase-3 antibody was purchased from Cell Signaling Technology (Cat. no. 9661S, Beverly, MA, USA) and the secondary antibody (Immunofluorescence Staining Kit with FITC-Labeled Goat Anti-Rabbit IgG, Cat. no. P0186) was purchased from Beyotime. The hemolymph sample $(20 \mu \mathrm{L})$ was mixed with $180 \mu \mathrm{L}$ of HBSS, allowed to stand, fixed, and washed with PBS as described above for TUNEL staining, and then $1 \mathrm{~mL}$ of cell permeabilization solution was added to it. The slide was allowed to stand for $60 \mathrm{~min}$ at room temperature and was then washed with PBS. Primary antibody of caspase- 3 antibody $(200 \mu \mathrm{L})$ was added dropwise and the slide was incubated in the dark overnight at $4{ }^{\circ} \mathrm{C}$ with shaking. After the slide was washed three times with PBS, $200 \mu \mathrm{L}$ of secondary antibody was added dropwise and incubated at $37{ }^{\circ} \mathrm{C}$ in the dark for $60 \mathrm{~min}$ on a shaker. After the slide was washed with PBS, Antifade Mounting Medium was added dropwise. The characteristic green fluorescence of the secondary antibody was observed.

\section{Quantitative real-time polymerase chain reaction}

The gene transcript levels were determined with the SYBR® Premix Ex Taq ${ }^{\mathrm{TM}}$ (Perfect Real Time) kit (TaKaRa, Dalian, China). The total reaction volume was $20 \mu \mathrm{L}$ and the gene primer sequences are given in Table S1. $\dagger$ The cycling conditions were: denaturation at $95{ }^{\circ} \mathrm{C}$ for $1 \mathrm{~min}$; followed by 45 cycles of $95{ }^{\circ} \mathrm{C}$ for $5 \mathrm{~s}, 55{ }^{\circ} \mathrm{C}$ for $10 \mathrm{~s}$, and $72{ }^{\circ} \mathrm{C}$ for $10 \mathrm{~s}$. The data were processed with Sequence Detection Software (version 1.3.1) in the instrument, and the efficiency was corrected according to the literature ${ }^{34}$ (three biological replicates).

\section{Results}

\section{Hemocyte counts}

The differences in the toxicity of the three types of nanomaterials administered at high doses to silkworm larvae were significant. At $96 \mathrm{~h}$ after exposure to CdTe QDs, the growth rate of the larvae was significantly lower than in the control group (60\% of the control rate), whereas there was no difference in the growth rates of the SiNP- and C-NCD-exposed groups and the control group (Fig. S1a-c $\dagger$ ). Within 96 h of exposure to CdTe QDs, $80 \%$ of the silkworms had died and the mortality rate was time dependent (Fig. S1d $\dagger$ ). No larval death was observed in the SiNP- or C-NCD-exposed group, or in the control group.

The hemolymph was observed with CLSM $24 \mathrm{~h}$ after the injection of the nanomaterial. The characteristic green fluorescence of the C-NCDs and CdTe QDs and the characteristic blue fluorescence of the SiNPs were detected in the hemocytes. Hemagglutination was also observed in the C-NCD- and CdTe-QDexposed groups, but was not observed in the SiNP-exposed group (Fig. 1a). These results indicate that the three types of nanomaterials administered via dorsal vein injection quickly entered the circulating hemocytes and caused differential hemagglutination reactions, suggesting that the responses of silkworm hemocytes to the different nanomaterials differed. Furthermore, after exposure to the nanomaterials, the morphology of the five types of hemocytes in the larval hemolymph became abnormal (Fig. 1b).

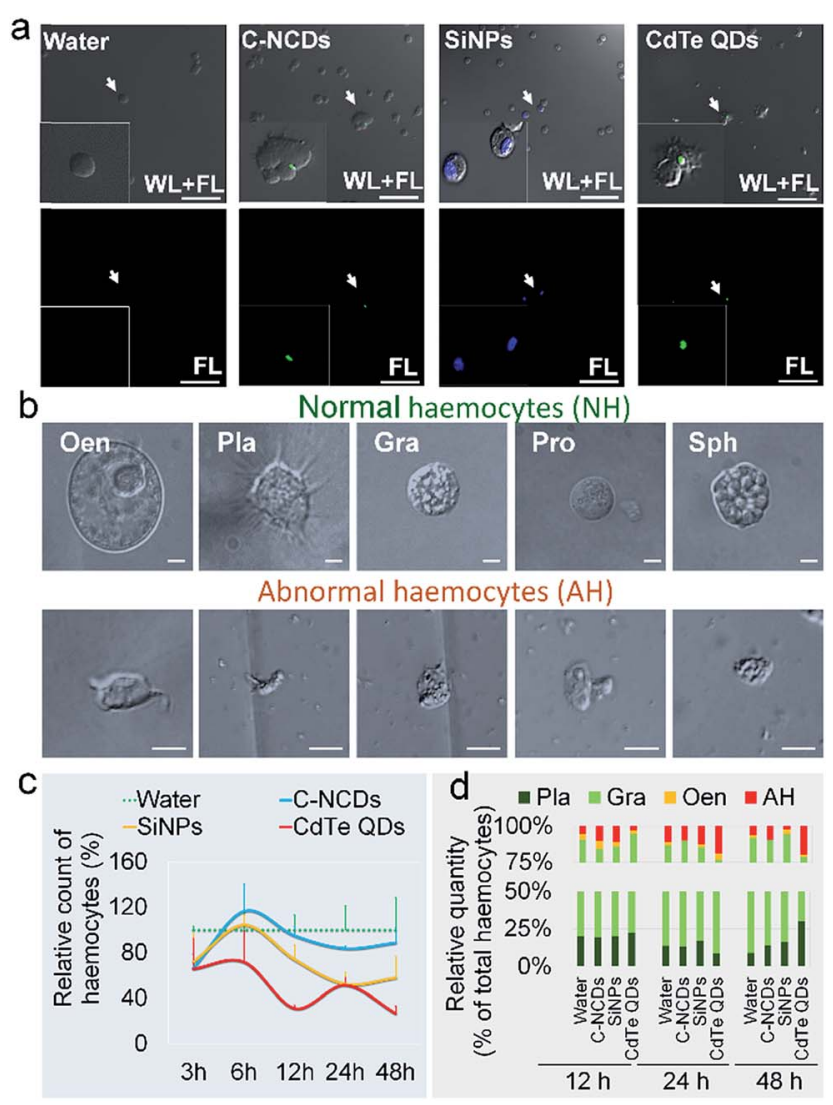

Fig. 1 Changes in hemolymphatic hemocytes after exposure to CdTe QDs, C-NCDs, or SiNPs. (a) Characteristic fluorescence of CdTe QDs, C-NCDs, and SiNPs monitored with confocal microscopy $24 \mathrm{~h}$ after injection. $\mathrm{FL}$, fluorescent images. $\mathrm{WL}+\mathrm{FL}$, merged white light and fluorescent images. (b) Morphology of normal hemocytes and abnormal hemocytes. (c) Relative circulating hemocyte counts. (d) Relative numbers of hemocyte types. Oen, oenocytoids; Pla, plasmacytes; Gra, granulocytes; Pro, prohacmocytes; Sph, spheroidocytes; $\mathrm{AH}$, abnormal hemocytes. Fifth-instar larvae were injected via the dorsal vein at $48 \mathrm{~h}$ old with $10 \mu \mathrm{L}$ of $32 \mu \mathrm{mol} \mathrm{L}{ }^{-1} \mathrm{CdTe}$ QDs, $0.39 \mu \mathrm{g} \mu \mathrm{L}^{-1}$ SiNPs, or $1 \mu \mathrm{g} \mu \mathrm{L}^{-1} \mathrm{C}-\mathrm{NCD}$. Sterilized purified water $(10 \mu \mathrm{L})$ was injected as the control. Triplicate larvae were tested each time. Bar $=50 \mu \mathrm{m}$. 
The results of the hemocyte counts (Fig. 1c) showed that $3 \mathrm{~h}$ after exposure to the three types of nanomaterials, the numbers of hemocytes were significantly reduced to about $65 \%$ of the number in the control group. The numbers of hemocytes in the C-NCD- and SiNP-exposed groups recovered rapidly, by $6 \mathrm{~h}$ after exposure. The number of hemocytes in the C-NCD-exposed group was restored to the control level at $48 \mathrm{~h}$ after exposure, whereas the number of hemocytes in the SiNP-exposed group was restored by $6 \mathrm{~h}$ after exposure, but decreased again and was significantly lower than that in the control group $48 \mathrm{~h}$ after exposure. It is noteworthy that the number of hemocytes in the CdTe-QD-exposed group did not recover to the control level $6 \mathrm{~h}$ after exposure, as occurred in the C-NCD- and SiNP-exposed groups, but decreased further, and was significantly lower than in the control group at $48 \mathrm{~h}$ after exposure. These results indicate that the effects of the three types of nanomaterials on the number of circulating hemocytes were significantly different.

To investigate the cause of the reduction in circulating hemocytes, we undertook a differential statistical analysis of the three most-abundant types of hemocytes in the hemolymph: granulocytes (Gra), oenocytoids (Oen), and plasmacytes (Pla), together with abnormal hemocytes $(\mathrm{AH})$. Fig. 1d shows that at $12 \mathrm{~h}$ after exposure to CdTe QDs, the hemocyte composition in the hemolymph had changed significantly, with an increased proportion of Gra. At $24 \mathrm{~h}$ after exposure, the proportions of Pla and Gra had decreased, whereas the proportion of $\mathrm{AH}$ had increased. At $48 \mathrm{~h}$ after exposure, the proportion of Pla was restored to the level in the control group, whereas the proportion of Gra was further reduced and the proportion of $\mathrm{AH}$ was still significantly higher than that in the control group. In the SiNP- and C-NCD-exposed groups, only the proportion of Gra decreased in the initial $12 \mathrm{~h}$, but was then restored to the level in the control group. These results suggest that the toxicity of the nanomaterials differs in different types of hemocytes.

The secretion of hemocytes by the HO is an important source of circulating hemocytes in the silkworm. Our previous studies have shown that CdTe QDs induce oxidative HO damage, leading to a dose- and time-dependent reduction in hemocyte secretion by the HO. ${ }^{20}$ Therefore, we proposed evaluating the hematopoietic toxicity of CdTe QDs in the silkworm by detecting the levels of ROS in the silkworm HO. ${ }^{20,33}$ In the present study, the results of ROS staining (Fig. S2 $\dagger$ ) showed that the green fluorescence intensity representing the ROS levels in the HO increased continuously in the larvae $48 \mathrm{~h}$ after exposure to CdTe QDs. However, the green fluorescence in the HO was weak in the SiNP- and C-NCD-exposed groups, and almost no characteristic ROS fluorescence was observed in the HO in the SiNPexposed group. Therefore, we consider that the reduced number of hemocytes in the hemolymph after exposure to SiNPs was not caused by reduced hemocyte secretion resulting from oxidative damage to the HO, but might be associated with the direct death of the hemocytes.

\section{Expression of immunogenesis-associated genes}

The differences in hemagglutination caused by exposure to the three types of nanomaterials (Fig. 1a) suggest that the effects of the different nanomaterials on the innate immune response in the hemolymph differed. The innate immune response is an acute response. Therefore, we analyzed the innate-immunityrelated genes encoding moricin (Mor) and cecropin A $(\operatorname{Cec} A),{ }^{35,36}$ the hemocyte-phagocytosis-related genes encoding cell death suppressor 6 (Ced-6), actin A1 (A1), and the gene encoding tetraspanin $\mathrm{E},{ }^{37}$ and the gene encoding the P38MAPK signal transduction protein ( $p 38)$, which plays an important role in the stress responses, including inflammation and apoptosis ${ }^{37,38}$ in the silkworm hemolymph. We investigated the changes in the expression of these six genes after exposure to the nanomaterials.

The results at $3 \mathrm{~h}$ after exposure (Fig. 2a-f) showed that the mRNA levels of Mor and $A 1$ were increased in the silkworm hemolymph in the C-NCD-exposed group, and the expression level of $p 38$ was also upregulated, whereas the mRNA levels of tetraspanin E, CecA, and Ced-6 were maintained at control group levels. This suggests that $\mathrm{C}-\mathrm{NCD}$ exposure triggers a rapid innate immune response in the silkworms and induces the upregulation of antimicrobial peptides, which results in the

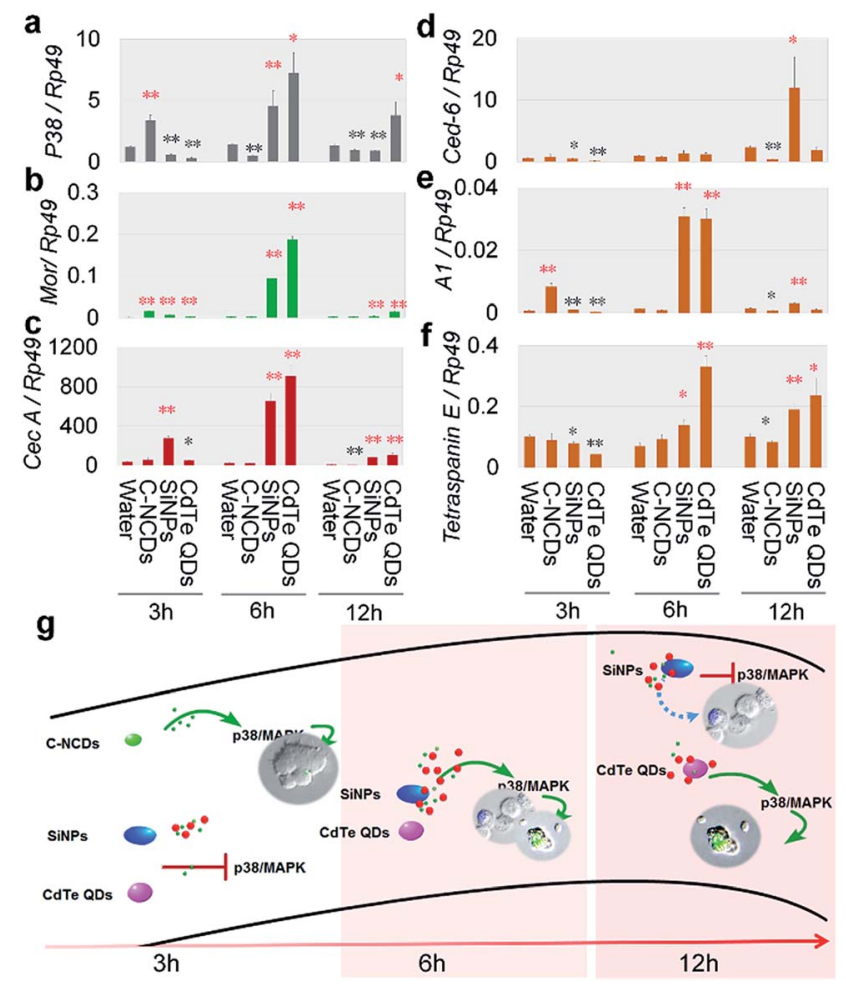

Fig. 2 Expression of immunity-associated genes after exposure to CdTe QDs, C-NCDs, or SiNPs. (a) p38 MAPK (p38), (b) moricin (Mor), (c) cecropin A (CecA), (d) cell death suppressor 6 (Ced-6), (e) actin A1 (A1), (f) tetraspanin E gene. (g) Difference pattern of the innate immune response in the hemolymph of silkworm larvae after exposure to CdTe QDs, C-NCDs, or SiNPs. Fifth-instar larvae were injected via the dorsal vein at $48 \mathrm{~h}$ old with $10 \mu \mathrm{L}$ of $32 \mu \mathrm{mol} \mathrm{L}{ }^{-1}$ CdTe QDs, $0.39 \mu \mathrm{g} \mu \mathrm{L}^{-1}$ SiNPs, or $1 \mu \mathrm{g} \mu \mathrm{L}^{-1} \mathrm{C}-\mathrm{NCD}$. Sterilized purified water $(10 \mu \mathrm{L})$ was used for the control injection. Hemolymph was collected from the larvae and qPCR performed at 3, 6, and $12 \mathrm{~h}$ after injection. Rp49 gene was used as the reference. $* P<0.05$ and $* * P<0.01$ versus the control group (repeated three times; every hemolymph sample contained equal volumes from three larvae). 
recognition and phagocytosis of C-NCDs by hemocytes, probably through the P38MAPK pathway. At $3 \mathrm{~h}$ after exposure to SiNPs, although the expression of Mor and CecA was upregulated, the transcription of $p 38$ was inhibited, and there was no evidence of enhanced hemocyte phagocytosis. At $3 \mathrm{~h}$ after exposure to CdTe QDs, only the expression of Mor was upregulated, and the expression of $p 38$ was downregulated.

Similar innate immune responses appeared at $6 \mathrm{~h}$ after exposure to SiNPs or CdTe QDs, when the expression of Mor, $C e c A, p 38, A 1$, and the tetraspanin $E$ gene was upregulated, indicating that the SiNPs and CdTe QDs were recognized and phagocytosed by the silkworm hemocytes (Fig. 2a-f). At $12 \mathrm{~h}$ after exposure (Fig. 2a-f), the transcript levels of these six genes in the CdTe-QD-exposed group showed the same pattern as at $6 \mathrm{~h}$ after exposure. However, in the SiNP-exposed group, the expression of Mor, CecA, A1, and the tetraspanin E gene was still elevated, whereas the mRNA level of $p 38$ was significantly downregulated, suggesting that although the silkworm hemocytes recognized and phagocytosed the CdTe QDs and SiNPs, their p38MAPK signaling pathway responses differed. It is noteworthy that the mRNA levels of $p 38$ were significantly downregulated at $6 \mathrm{~h}$ and $12 \mathrm{~h}$ after exposure to C-NCDs (Fig. 2a-f), and that the transcription levels of Mor, CecA, A1, and the tetraspanin $E$ gene were either maintained at the level of the control group or downregulated, suggesting that not only was the innate immune response (including phagocytosis) not activated in the hemolymph in this period, but that it was also downregulated. The differences in the effects of the three types of nanomaterials on the expression of the immune-related genes in the hemolymph reflect differences in rates and intensities of their recognition and clearance by hemocytes, and differences in the mechanisms involved. Based on these results, we have summarized the different patterns of the innate immune response to the three types of nanomaterials in silkworm hemocytes in Fig. 2g.

\section{Differences in mechanisms of hemocyte death induced by three types of nanomaterials}

The details of the programmed cell death (PCD) induced are a reliable indicator of the toxicity of nanomaterials. Therefore, in this study, the differences in the toxicity of the three types of nanomaterials were compared based on the details of hemocyte autophagy, apoptosis, and necrosis.

Necrotic hemocytes were detected with the classical Hoechst/ PI staining method. In the CdTe-QD-exposed group, a large number of hemocyte nuclei were dyed red by PI, and these hemocytes were confirmed with Hoechst staining. However, only a small number of hemocyte nuclei were dyed red by PI in the C-NCD- and SiNP-exposed groups (Fig. 3a).

A further quantitative analysis (Fig. 3b) showed that the number of Hoechst/PI-positive hemocytes increased sharply at $12 \mathrm{~h}$ after exposure to CdTe QDs compared with the number in the control group, reaching $44.6 \%$ of the total number of hemocytes. At $48 \mathrm{~h}$ after exposure, the positive hemocytes still remained at a high level. At 12,24 , and $48 \mathrm{~h}$ after exposure to SiNPs or C-NCDs, although Hoechst/PI double staining
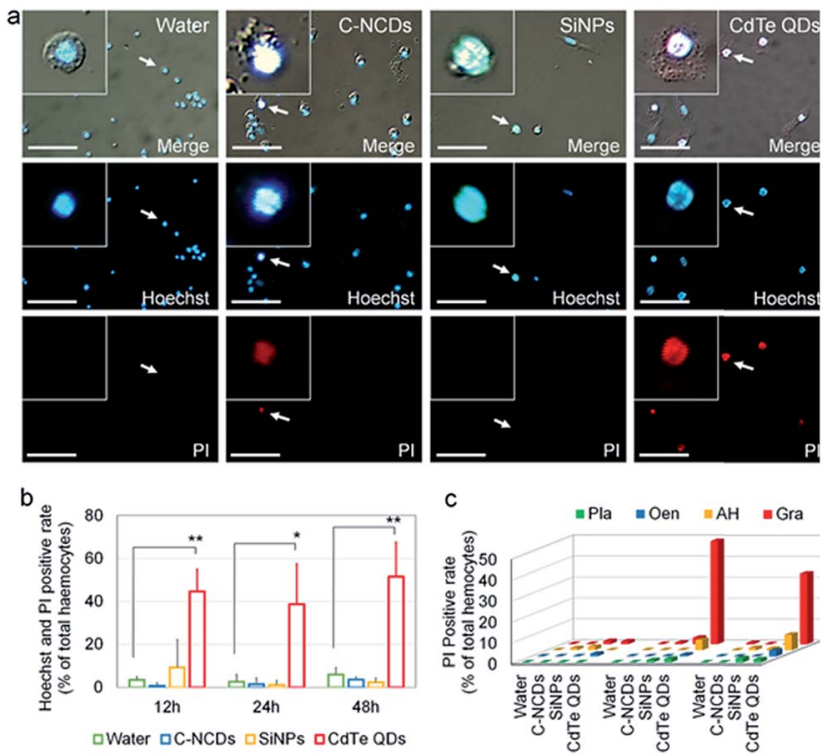

Fig. 3 Survey of hemocyte necrosis. (a) Fluorescent images of Hoechst/propidium iodide (PI)-stained hemocytes $24 \mathrm{~h}$ after nanomaterial injection. Pl, fluorescent images stained with PI. Hoechst, fluorescent images stained with Hoechst. Merge, merged white light and Hoechst/PI-stained fluorescent images. (b) Hoechst/PI-positive rate in hemocytes. (c) Number of PI-positive hemocyte types. Pla, plasmacytes; Gra, granulocytes; Oen, oenocytoids; AH, abnormal hemocytes. Fifth-instar larvae were injected via the dorsal vein at $48 \mathrm{~h}$ old with $10 \mu \mathrm{L}$ of $32 \mu \mathrm{mol} \mathrm{L}{ }^{-1}$ CdTe QDs, $0.39 \mu \mathrm{g} \mu \mathrm{L}^{-1}$ SiNPs, or $1 \mu \mathrm{g} \mu \mathrm{L}^{-1} \mathrm{C}-\mathrm{NCD}$. Sterilized purified water $(10 \mu \mathrm{L})$ was used for the control injection. Hemolymph was collected from the larvae and stained with Hoechst/PI at 12, 24, and $48 \mathrm{~h}$ after injection. $* P<0.05$ and $* * P<$ 0.01 versus the control group (repeated three times; every hemolymph sample contained equal volumes from three larvae). Bar $=50 \mu \mathrm{m}$.

detected positive hemocytes, the proportion of positive hemocytes did not differ significantly between the exposed groups and the control group. Therefore, in this study, the exposure of silkworm larvae to high doses of SiNPs or C-NCDs did not increase hemocyte necrosis.

Because PI single staining has little effect on the morphology of silkworm hemocytes, we counted the different types of PIpositive hemocytes. The results (Fig. 3c) showed that the PIpositive necrotic hemocytes in the CdTe-QD-exposed group were mainly Gra and $\mathrm{AH}$, and that PI-positive necrotic Gra accounted for $49.1 \%$ of the circulating hemocytes at $24 \mathrm{~h}$ after exposure. Although the proportion of Gra decreased to $33.7 \%$ at $48 \mathrm{~h}$ after exposure, the proportion of $\mathrm{AH}$ in the circulating hemocytes in the hemolymph increased from $5 \%$ to $7.4 \%$ at $24 \mathrm{~h}$ after exposure. Therefore, we infer that the CdTe QDs that enter the circulating hemocytes in the hemolymph not only induce hemocyte necrosis, but also deform the necrotic hemocytes.

Staining autophagic vacuoles with MDC is considered a reliable method of assessing the degree of autophagy. ${ }^{39}$ In this study, MDC staining showed that the formation of autophagic vacuoles in the circulating hemocytes in the hemolymph differed significantly after exposure to the different nanomaterials. The degree of autophagic vacuole formation was 
significantly higher in the C-NCD- and SiNP-exposed groups than in the CdTe-QD-exposed group (Fig. 4a). The hemocyte counts (Fig. 4c) showed that at 12-48 h after exposure to CdTe QDs, there was no significant difference in the proportion of MDC-positive cells among the total hemocytes.

Compared with the control group. However, $24 \mathrm{~h}$ after exposure to SiNPs, the proportion of MDC-positive hemocytes was significantly increased, although at $48 \mathrm{~h}$ after exposure, it had returned to the level in the control group. At $12 \mathrm{~h}$ after exposure to C-NCDs, the number of MDC-positive hemocytes was already significantly higher than in the control group, and the proportion of MDC-positive hemocytes continued to increase at $24 \mathrm{~h}$ and $48 \mathrm{~h}$ after exposure, when it was significantly higher than in the CdTe-QD- or SiNP-exposed group. These results indicate that the CdTe QDs did not induce obvious autophagy in the hemocytes at 12-48 h after exposure; and although the SiNPs induced autophagy in some hemocytes, they self-repaired before $48 \mathrm{~h}$. However, the C-NCDs timedependently induced severe autophagy in the hemocytes at 12-48 $\mathrm{h}$ after exposure, indicating that C-NCDs exert a specific toxic effect on hemocytes, although they do not cause hemocyte necrosis or obviously reduce the number of hemocytes.

MDC staining of autophagic vacuoles can directly reflect whether autophagy appear in the cells. ${ }^{39}$ To confirm the MDC staining results, we investigated the transcription levels of autophagy associated gene 6 (Atg6) and Atg 8 in the hemolymph, which are considered key genes in the regulation of autophagy in silkworm cells. ${ }^{\text {40-42 }}$ The expression of Atg6 and Atg8 was significantly upregulated at 12-48 h after exposure (Fig. $4 \mathrm{c} \& \mathrm{~d}$ ). It is noteworthy that although the transcript levels of these two key genes were upregulated in the CdTe-QD-exposed group,
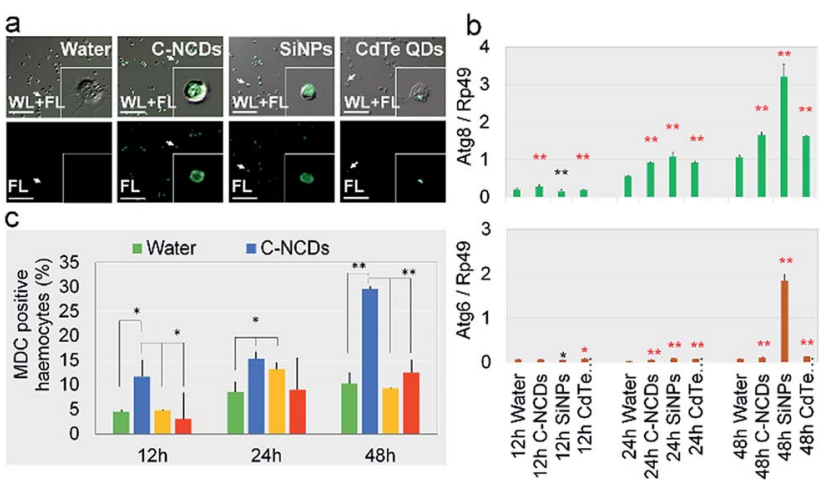

Fig. 4 Survey of hemocyte autophagy. (a) Fluorescent monodansylcadaverine (MDC)-stained images at $24 \mathrm{~h}$ after injection. $\mathrm{FL}$, fluorescent images stained with MDC. WL + FL, merged white light and MDC-stained fluorescent images. (b) MDC-positive rate in hemocytes. (c) Relative gene expression levels of autophagy-associated genes. Fifth-instar larvae were injected via the dorsal vein at $48 \mathrm{~h}$ old with 10 $\mu \mathrm{L}$ of $32 \mu \mathrm{mol} \mathrm{L}{ }^{-1}$ CdTe QDs, $0.39 \mu \mathrm{g} \mu \mathrm{L}^{-1}$ SiNPs, or $1 \mu \mathrm{g} \mu \mathrm{L}^{-1} \mathrm{C}-\mathrm{NCDs}$. Sterilized purified water $(10 \mu \mathrm{L})$ was used for the control injection. Hemolymph was collected from the larvae and stained with MDC or subjected to $\mathrm{qPCR}$ at 12,24 , and $48 \mathrm{~h}$ after injection. $* P<0.05$ and $* * P$ $<0.01$ versus the control group (repeated three times; every hemolymph sample contained equal volumes from three larvae). Bar = $100 \mu \mathrm{m}$ there was no significant difference in the degree of autophagic vacuole formation-which denotes autophagy-in these hemocytes compared with the control group (Fig. 4a \& b). This suggests that the hemocyte death caused by CdTe QD exposure does not result from cell autophagy. In contrast, although the transcription levels of Atg6 and Atg 8 were significantly higher $48 \mathrm{~h}$ after exposure to SiNPs than at an earlier time, and even higher than that in the C-NCD-exposed group at the same time, there were fewer MDC-positive hemocytes at $48 \mathrm{~h}$ than at $24 \mathrm{~h}$, and fewer than in the C-NCD-exposed group at the same time. This indicates that the increased autophagy signal in the SiNPexposed group was closely associated with cell repair.

Combining the results shown in Fig. 4, there is considerable discrepancy between results of MDC staining and the transcription levels of autophagy associated gene. In some cases, autophagy is induced by nanoparticles may be concealed by apoptosis, and the gene expression level of autophagy associated gene 6 (Atg6) and Atg8 are vulnerable to interfere by other factors. ${ }^{59}$ Hence, we speculated that the cell autophagy induced by CdTe QDs or SiNPs is masked by the emergence of other forms of PCD, so we focused our investigation on the apoptosis of the hemocytes.

The formation of apoptotic hemocytes, stained with TUNEL, at $24 \mathrm{~h}$ after exposure showed that the degree of hemocyte apoptosis in the CdTe-QD- or SiNP-exposed group was significantly higher than in the C-NCD-exposed group (Fig. 5a). Hemocyte counting (Fig. 5b) showed that in the CdTe-QDexposed group, the TUNEL-positive rate (TPR) in the hemocytes
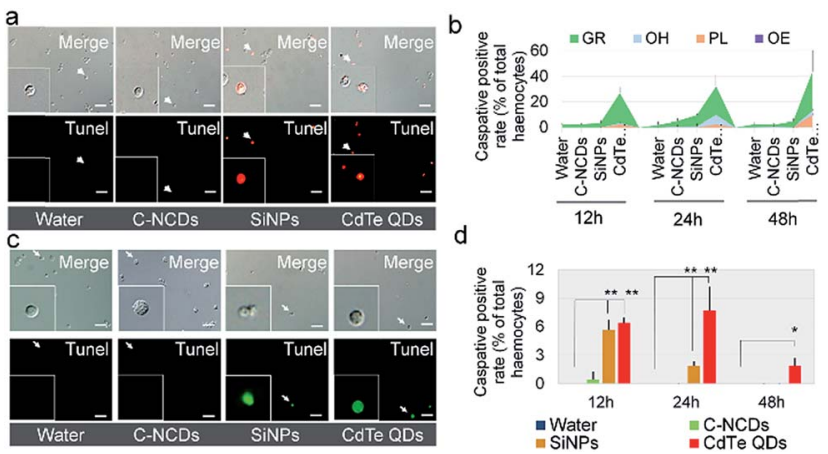

Fig. 5 Survey of hemocyte apoptosis. (a) Fluorescent images of TdTmediated dUTP-nick end labeling (TUNEL) staining of hemocytes $24 \mathrm{~h}$ after injection. (b) TUNEL-positive rate in hemocytes and their subtypes. (c) Caspase-3-immunofluorescent images of hemocytes $24 \mathrm{~h}$ after injection. Pla, plasmacytes; Gra, granulocyte; Oen, oenocytoids; AH, abnormal hemocytes. (d) Caspase-3-immunofluorescence-positive rate (CPR) of hemocytes. Fifth-instar larvae were injected via the dorsal vein at $48 \mathrm{~h}$ old with $10 \mu \mathrm{L}$ of $32 \mu \mathrm{mol} \mathrm{L}{ }^{-1} \mathrm{CdTe}$ QDs, $0.39 \mu \mathrm{g} \mu \mathrm{L}^{-1}$ SiNPs, or $1 \mu \mathrm{g} \mu \mathrm{L}^{-1} \mathrm{C}-\mathrm{NCD}$. Sterilized purified water $(10 \mu \mathrm{L})$ was used for the control injection. Hemolymph was collected from the larvae and stained with TUNEL or immunohistochemically for caspase- 3 at 12,24 , and $48 \mathrm{~h}$ after injection. TUNEL, fluorescent images stained with TUNEL. Caspase, fluorescent images immunohistochemically stained for caspase-3. Merge, merged white light and TUNEL- (a) or caspase-3-stained (c) fluorescent images. ${ }^{*} P<$ 0.05 and $* * P<0.01$ versus the control group (repeated three times; every hemolymph sample contained equal volumes from three larvae). Bar $=100 \mu \mathrm{m}$. 
increased to $32 \%$ of the total number of hemocytes in the hemolymph at $12 \mathrm{~h}$ after exposure, and TPR continued to increase with time after exposure, reaching $44 \%$ at $24 \mathrm{~h}$ and $63 \%$ at $48 \mathrm{~h}$, thus displaying a time-dependent effect. At $12 \mathrm{~h}$ after exposure to SiNPs, there was no significant difference in TPR between the exposed group and the control group, but at $24 \mathrm{~h}$ after exposure, TPR reached $10 \%$, which was significantly different from the control group. However, at $48 \mathrm{~h}$ after exposure, TPR had returned to the normal level in the control group, indicating that although the high-dose SiNPs used in this study induced apoptosis in silkworm hemocytes, the hemocytes quickly returned to normal. At 12, 24, and $48 \mathrm{~h}$ after exposure to C-NCDs, there was no significant difference in TPR between the exposed group and the control group, so we inferred that $\mathrm{C}$ NCDs exposure does not cause apoptosis in silkworm hemocytes. When the TUNEL-positive hemocytes were counted (Fig. 5b), they were mainly Gra and AH in both the CdTe-QD- and SiNP-exposed groups, but a certain proportion of Pla was also present in the CdTe-QD-exposed group.

An immunohistochemical analysis of the executive apoptotic protein caspase-3 (Fig. 5c) supported the TUNEL staining results. Immunofluorescence for caspase- 3 protein was detected in the apoptotic hemocytes $24 \mathrm{~h}$ after exposure to the nanomaterials, and was more severe in the CdTe-QD- and SiNPexposed groups than in the C-NCD-exposed group (Fig. 5c). Hemocyte counting showed no significant difference in the caspase-3-positive rate (CPR) in the hemocytes (Fig. 5d) at 12, 24 , or $48 \mathrm{~h}$ after $\mathrm{C}-\mathrm{NCD}$ exposure compared with the control group. Therefore, we inferred that C-NCD exposure does not cause apoptosis in silkworm hemocytes. At $12 \mathrm{~h}$ after exposure to SiNPs, CPR was significantly higher than in the control group; at $24 \mathrm{~h}$ after exposure, although CPR was still higher

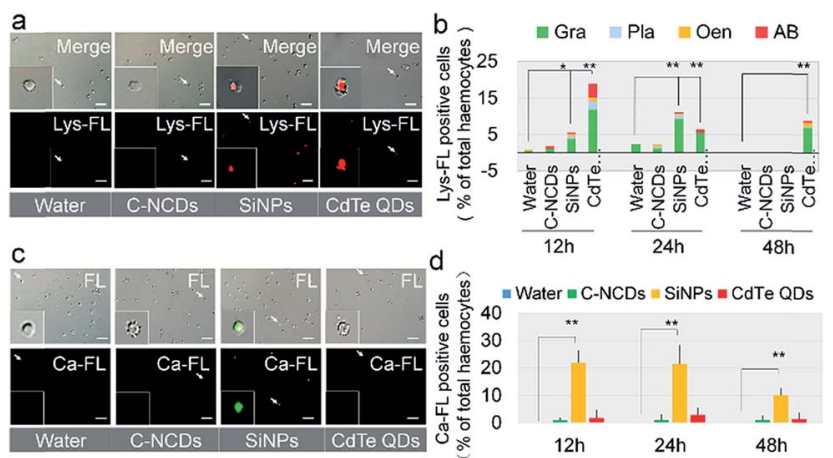

Fig. 6 Lysosome staining and fluorescent probing for calcium ions. (a) Images of fluorescently stained lysosomes (Lys-FL) and (c) fluorescently stained calcium ions ( $\mathrm{Ca}-\mathrm{FL}$ ) in hemocytes at $24 \mathrm{~h}$ after injection. (b) Lys-FL-positive rates in hemocytes and their subtypes. Pla, plasmacytes; Gra, granulocyte; Oen, oenocytoids; AH, abnormal hemocytes. (d) Ca-FL-positive rates of hemocytes. Fifth-instar larvae were injected via the dorsal vein at $48 \mathrm{~h}$ old with $10 \mu \mathrm{L}$ of $32 \mu \mathrm{mol} \mathrm{L}{ }^{-1}$ CdTe QDs, $0.39 \mu \mathrm{g} \mu \mathrm{L}^{-1}$ SiNPs, or $1 \mu \mathrm{g} \mu \mathrm{L}^{-1} \mathrm{C}-\mathrm{NCD}$. Sterilized purified water $(1 \mu \mathrm{L})$ was used for the control injection. Hemolymph was collected from the larvae and stained for lysosomes or fluorescently probed for calcium at 12,24 , and $48 \mathrm{~h}$ after injection. ${ }^{*} P<$ 0.05 and $* * P<0.01$ versus the control group (repeated three times, and every hemolymph sample contained equal volumes from three larvae). Bar $=100 \mu \mathrm{m}$. than in the control group, it had decreased significantly; CPR continued to decrease and had returned to the level of the control group at $48 \mathrm{~h}$ after exposure. This also indicates that although the high-dose SiNPs used in this study induced shortterm apoptosis in the silkworm hemocytes, the hemocytes selfrepaired. At $12 \mathrm{~h}$ after exposure to CdTe QDs, CPR was significantly higher than in the control group; it was even higher at $24 \mathrm{~h}$ after exposure, but had decreased at $48 \mathrm{~h}$, although it was still significantly higher than in the control group.

The results described above show that hemocytes became apoptotic after exposure to CdTe QDs or SiNPs. Further investigation of the lysosomal apoptotic signalling pathway showed that the signal intensity of the pathway was significantly different in the hemocytes at $24 \mathrm{~h}$ after exposure to the three types of nanomaterials, and the degree of lysosome-mediated apoptosis in the hemocytes was significantly higher in the CdTe-QD- and SiNP-exposed groups than in the C-NCD-exposed group (Fig. 6a). A quantitative analysis (Fig. 6b) showed that $18 \%$ of circulating hemocytes were positive for lysosomal staining at $12 \mathrm{~h}$ after exposure to CdTe QDs, and the proportion was significantly higher than in the control group at $48 \mathrm{~h}$. The proportion of lysosome-positive hemocytes also increased in the SiNP-exposed group at 12 and $24 \mathrm{~h}$ after exposure, but had returned to the level in the control group at $48 \mathrm{~h}$ after exposure. There was no significant difference in the proportion of lysosome-positive hemocytes at $48 \mathrm{~h}$ after exposure to C-NCDs and in the control group. These results indicate that the ability of the three types of nanomaterials to induce the lysosomal apoptotic signalling pathway in silkworm hemocytes differs significantly. The differential counts of hemocytes (Fig. 6b) showed that in both the CdTe-QD- and SiNP-exposed groups, the positively lysosome-stained apoptotic hemocytes were mainly Gra, followed by Pla and Oen. The two latter cell types are directly involved in phagocytosis during the cellular immune response.

Investigation of the endoplasmic reticulum/calcium ion apoptotic signalling pathway (Fig. 6c \& d) showed that at $12 \mathrm{~h}$ after exposure to SiNPs, the intracellular calcium ion level had increased significantly and was maintained at this level until $24 \mathrm{~h}$ after exposure. It had decreased by $48 \mathrm{~h}$ after exposure, but was still significantly higher than in the control group. Interestingly, there was no significant difference between the intracellular calcium ion levels in the CdTe-QD-exposed group and the control group or the C-NCD-exposed group. These results suggest that SiNPs induce apoptosis in hemocytes through the endoplasmic reticulum/calcium ion apoptotic signalling pathway, whereas CdTe QDs do not rely on this pathway, although they cause the apoptosis of large numbers of hemocytes.

\section{Discussion}

\section{Three types of nanomaterials display different biological toxicity}

CdTe QDs $^{\mathbf{4 3 - 4 5}}$ SiNPs $^{3,4}$ and C-NCDs ${ }^{46,47}$ are representative nanomaterials which have potentially broad applications in biomedical fields based on their characteristics. Previous studies have suggested that CdTe QDs are strongly toxic to nuclei and that 
high-dose exposure can lead to clearly irreversible damage to organisms, with high mortality rates..$^{\mathbf{2 0 , 3 2 , 4 8}}$ The biological toxicity of SiNPs is still controversial, and some studies have shown that SiNPs are biocompatible nanomaterials with low toxicity or even no toxicity at both the cellular and organismal levels., ${ }^{3,49}$ Liu et $a .^{50}$ reported that although SiNPs showed no toxicity in monkeys, they caused liver damage and hemolysis in mice. Several reports have also clearly stated that SiNPs induce ROS formation in cells, initiate cell autophagy and apoptosis, ${ }^{\mathbf{5 1}}$ and are biologically toxic, ${ }^{52}$ although the organisms can self-repair. ${ }^{33}$ C-NCDs are considered to be a low-toxicity material with good biocompatibility and many potential applications. ${ }^{\mathbf{4 6}}$

No study has previously compared the mechanisms of toxicity of CdTe QDs, SiNPs, and C-NCDs in animal hemocytes. In this study, silkworm larvae were exposed to high-dose CdTe QDs, SiNPs, or C-NCDs. Only CdTe QDs showed adverse effects on weight gain and vitality, whereas SiNPs and C-NCDs showed only temporary and self-repairing effects or even no adverse effects. The three types of nanomaterials that entered the silkworm hemolymph were engulfed by hemocytes, reducing the numbers of hemocytes within $48 \mathrm{~h}$ to varying degrees. Among these nanomaterials, CdTe QDs had the greatest effect, whereas CNCDs only had only a weak adverse effect. CdTe QDs directly damaged the circulating hemocytes, and destroyed the secretion of hemocytes by the HO by inducing oxidative HO damage. However, no oxidative HO damage was observed after exposure to SiNPs or C-NCDs. The three types of nanomaterials also showed significant differences in their toxicity to different types of circulating hemocytes, and Gra, Pla, and Oen, which are directly involved in the blood immune response, surrounding and phagocytosing exogenous substances, were clearly damaged.

\section{Different toxicity mechanisms of three types of nanomaterials in hemocytes}

Cell death occurs primarily through PCD in animals. ${ }^{53}$ Cell autophagy can induce apoptosis, ${ }^{40}$ and even necrosis. ${ }^{54,55}$ Reports have shown that there were different types of cell death caused by nanomaterials, including autophagy, apoptosis, and necrosis. ${ }^{56}$ Multiple induction mechanisms involving lysosomes, ${ }^{57}$ the endoplasmic reticulum/calcium ions, ${ }^{58}$ the mitochondria, ${ }^{57}$ and reactive oxygen species (ROS) ${ }^{20}$ also exist. The toxic effects of the same nanomaterial can differ in different organisms or cells, causing discrepancies and contention across studies. ${ }^{59,60}$ Therefore, it is necessary to select a suitable experimental animal platform with which to investigate the types of programmed cell death caused by nanomaterials.

The results of the present study show that the PCD processes and patterns in silkworm hemocytes induced by three types of nanomaterials were very different. SiNPs exposure caused hemocyte autophagy, and also induced the apoptosis of hemocytes via two apoptotic pathways, the lysosomal and endoplasmic reticulum/calcium ion pathways, but no increase in necrotic hemocytes was observed. It is noteworthy that at $48 \mathrm{~h}$ after exposure to SiNPs, self-repair occurred in both the autophagic and apoptotic hemocytes, which may be related to the degradation of the SiNPs in the organisms and their secretion in the form of silicates. ${ }^{61}$ After exposure to C-NCDs, only autophagy occurred in the hemocytes, and no increase in apoptotic or necrotic hemocytes was observed, but the effects of C-NCDs did not decrease during the experimental period, which may be attributable to their resistance to degradation in this organism. $^{62}$

In this study, exposure to CdTe QDs induced lysosomalpathway-dependent apoptosis in the hemocytes and the rapid necrosis of large numbers of hemocytes. We observed no increase in endoplasmic reticulum/calcium ion apoptosis in the hemocytes, or even hemocyte autophagy. It has been reported that CdTe QDs enter cells by endocytosis and form phagosomes, which fuse with lysosomes. They are degraded to some extent by the acidic environment of the lysosome, ${ }^{63}$ resulting in the release of cytotoxic cadmium ions, ${ }^{\mathbf{6 4}}$ which induce strong apoptosis. A study in Saccharomyces cerevisiae showed that the severe apoptosis and rapid necrosis caused by CdTe QDS inhibited the autophagy of the cells. ${ }^{59}$ Therefore, we speculate that CdTe-QD-induced hemocyte autophagy might be masked by more-severe apoptosis and even necrosis because we did not analyze samples collected before $12 \mathrm{~h}$ after exposure in this

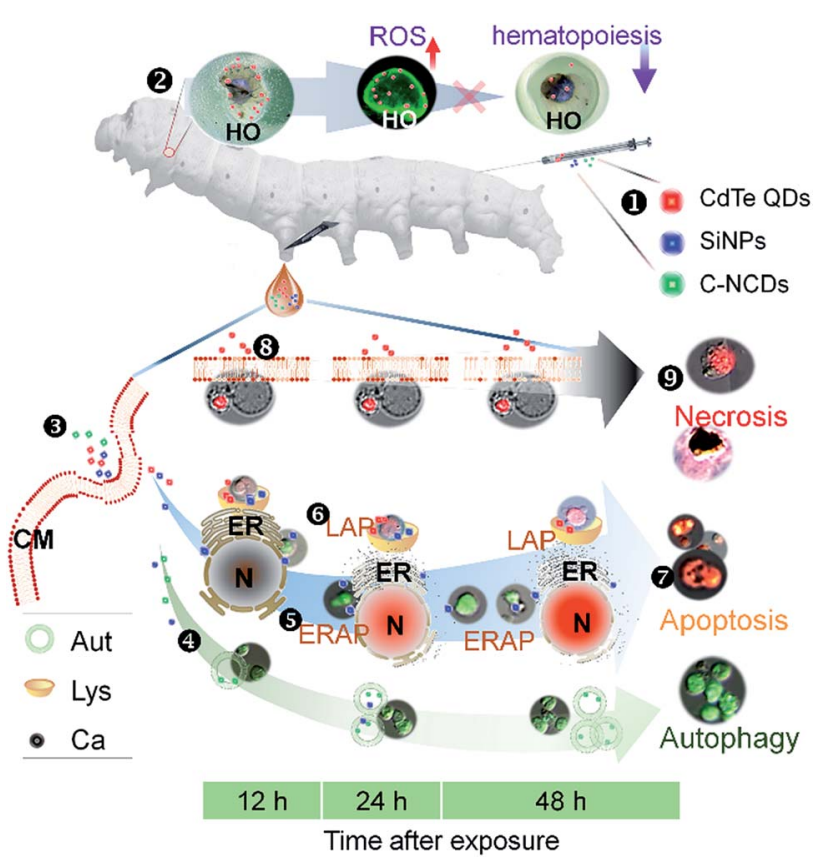

Fig. 7 Different patterns of programmed cell death induced by $\mathrm{C}-$ NCDs, SiNPs, and CdTe QDs in silkworm hemocytes. (1 Larvae were injected via the dorsal vein with C-NCDs, SiNPs, or CdTe QDs. (2) Exposure to CdTe QDs reduced the hematopoietic function of the hematopoietic organ $(\mathrm{HO})$ via the induced production of reactive oxygen species (ROS). (3 Nanomaterials entered the hemocytes via endocytosis. (4) Autophagy of hemocytes was caused by C-NCDs or SiNPs via the induced production of autophagosomes (Aut) in the cytoplasm. 6 Endoplasmic reticulum apoptotic pathway (ERAP) was triggered by SiNPs, releasing calcium ions (Ca) from the endoplasmic reticulum (ER). 6 Lysosomal apoptotic pathway (LAP) triggered by SiNPs or CdTe QDs induced the production of lysosomes (Lys). $\boldsymbol{\nabla}$ Apoptosis of hemocytes. 8 Necrosis of hemocytes caused by CdTe QDs by the disruption of the cytomembrane of the hemocytes (CM). $\boldsymbol{9}$ Necrosis of hemocytes. 
study. The rapid necrosis of hemocytes may be related to the cytotoxicity of the cadmium ions released by the lysosomal degradation of the quantum dots during apoptosis.

The immune systems of humans and other animals recognize and remove exogenous substances from the body, including drugs such as nanomaterials. ${ }^{65,66}$ Exploring the interactions between nanomaterials and the immune system will facilitate the development and application of new nanomaterials with improved biocompatibility.$^{67,68}$ The results of this study show that C-NCDs rapidly unregulated the expression of antimicrobial peptides in silkworm hemocytes, resulting in the recognition and phagocytosis of the C-NCDs by the p38MAPK pathway. After exposure to SiNPs or CdTe QDs, the expression of antimicrobial peptides in the haemocytes and their response to the p38MAPK pathway were slower than after C-NCD exposure, the response levels were higher, and the recognition and phagocytosis of the nanoparticles by the hemocytes was stronger. The mechanisms of the innate immune responses to SiNPs and CdTe QDs differed, and hemocyte recognition and phagocytosis may not depend on the p38MAPK pathway after SiNP exposure. Fig. 7 summarizes the differences in the toxicity mechanisms in hemocytes after silkworm larvae were exposed to CdTe QDs, SiNPs, or C-NCDs. CNCDs only caused hemocyte autophagy, but the effect lasted a long time. Exposure to both SiNPs and CdTe QDs caused hemocyte apoptosis, but the apoptotic mechanisms differed. CdTe QDs caused hemocyte apoptosis through the lysosomal pathway, whereas SiNPs also caused hemocyte apoptosis through the endoplasmic reticulum pathway.

\section{Conclusions}

Exposure to CdTe QDs, SiNPs, or C-NCDs exerted significantly different toxic effects on silkworm hemocytes via the induction of different PCD mechanisms and processes.

\section{Conflicts of interest}

There are no conflicts to declare.

\section{Acknowledgements}

This work was supported by the National Natural Science Foundation of China (No. 31672492 and 31472149), Provincial Key R \& D Program of Jiangsu [Project No. BE2015317], Priority Academic Program Development of Jiangsu Higher Education Institutions (PAPD), and the China Agriculture Research System (CARS; Grant no. CARS-22). The funders had no role in the study design, data collection and analysis, decision to publish, or preparation of the manuscript.

\section{References}

1 X. Yang, Z. Wang, Z. Xiang, D. Li, Z. Hu, W. Cui, L. Geng and Q. Fang, Peptide probes derived from pertuzumab by molecular dynamics modeling for HER2 positive tumor imaging, PLoS Comput. Biol., 2017, 13, DOI: 10.1371/ journal.pcbi.1005441.

2 H. Dong, Z. Liu, H. Zhong, H. Yang, Y. Zhou, Y. Hou, J. Long, J. Lin and Z. Guo, Melanin-associated synthesis of sers-active nanostructures and the application for monitoring of intracellular melanogenesis, Nanomaterials, 2017, 7, DOI: 10.3390/nano7030070.

$3 \mathrm{~W}$. H. Zhang, X. X. Hu and X. B. Zhang, Dye-doped fluorescent silica nanoparticles for live cell and in vivo bioimaging, Nanomaterials, 2016, 6, 81.

4 G. V. Deodhar, M. L. Adams and B. G. Trewyn, Controlled release and intracellular protein delivery from mesoporous silica nanoparticles, Biotechnol. J., 2017, 12, DOI: 10.1002/ biot.201600408.

5 P. Zhang, Y. C. Chiu, L. H. Tostanoski and C. M. Jewell, Polyelectrolyte multilayers assembled entirely from immune signals on gold nanoparticle templates promote antigen-specific T cell response, ACS Nano, 2015, 9, 64656477.

6 J. Lin, Y. Li, Y. Li, H. Wu, F. Yu, S. Zhou, L. Xie, F. Luo, C. Lin and Z. Hou, Drug/dye-loaded, multifunctional peg-chitosaniron oxide nanocomposites for methotraxate synergistically self-targeted cancer therapy and dual model imaging, ACS Appl. Mater. Interfaces, 2015, 7, 11908-11920.

7 M. M. Heidari, D. Asgari, J. Barar, H. Valizadeh, V. Kafil, A. Abadpour, E. Moumivand, J. S. Mojarrad, M. R. Rashidi, G. Coukos and Y. Omidi, Tamoxifen loaded folic acid armed PEGylated magnetic nanoparticles for targeted imaging and therapy of cancer, Colloids Surf., B, 2013, 106, 117-125.

8 M. Mahmoudi, K. Azadmanesh, M. A. Shokrgozar, W. S. Journeay and S. Laurent, Effect of nanoparticles on the cell life cycle, Chem. Rev., 2011, 111, 3407-3432.

9 B. G. Kim, P. H. Lee, S. H. Lee, M. K. Park and A. S. Jang, Effect of $\mathrm{TiO}_{2}$ nanoparticles on inflammasome-mediated airway inflammation and responsiveness, Allergy, Asthma Immunol. Res., 2017, 9, 257-264.

10 S. Chen, R. Yin, K. Mutze, Y. Yu, S. Takenaka, M. Königshoff and T. Stoeger, No involvement of alveolar macrophages in the initiation of carbon nanoparticle induced acute lung inflammation in mice, Part. Fibre Toxicol., 2016, 13, 33.

11 K. P. Wen, Y. C. Chen, C. H. Chuang, H. Y. Chang, C. Y. Lee and N. H. Tai, Accumulation and toxicity of intravenouslyinjected functionalized graphene oxide in mice, J. Appl. Toxicol., 2015, 35, 1211-1218.

12 S. Gurunathan, J. W. Han, V. Eppakayala and J. H. Kim, Biocompatibility of microbially reduced graphene oxide in primary mouse embryonic fibroblast cells, Colloids Surf., B, 2013, 105, 58-66.

13 S. K. Singh, M. K. Singh, M. K. Nayak, S. Kumari, S. Shrivastava, J. J. Grácio and D. Dash, Thrombus inducing property of atomically thin graphene oxide sheets, ACS Nano, 2011, 5, 4987-4996.

14 I. Pujalté, D. Dieme, S. Haddad, A. M. Serventi and M. Bouchard, Toxicokinetics of titanium dioxide (TiO2) nanoparticles after inhalation in rats, Toxicol. Lett., 2017, 265, 77-85. 
15 E. Fröhlich, Value of phagocyte function screening for immunotoxicity of nanoparticles in vivo, Int. J. Nanomed., 2015, 10, 3761-3778.

16 T. Zhang, M. Tang, S. Zhang, Y. Hu, H. Li, T. Zhang, Y. Xue and $\mathrm{Y}$. Pu, Systemic and immunotoxicity of pristine and PEGylated multi-walled carbon nanotubes in an intravenous 28 days repeated dose toxicity study, Int. J. Nanomed., 2017, 12, 1539-1554.

$17 \mathrm{~V}$. Baumans, Use of animals in experimental research: an ethical dilemma?, Gene Ther., 2004, 1, S64-S66.

18 L. M. Stuart and R. A. Ezekowitz, Phagocytosis and comparative innate immunity: learning on the fly, Nat. Rev. Immunol., 2008, 8, 131-141.

19 A. Blagodatski, A. Sergeev, M. Kryuchkov, Y. Lopatina and V. L. Katanaev, Diverse set of Turing nanopatterns coat corneae across insect lineages, Proc. Natl. Acad. Sci. U. S. A., 2015, 112, 10750-10755.

20 T. Liu, R. Xing, Y. F. Zhou, J. Zhang, Y. Y. Su, K. Q. Zhang, Y. He, Y. H. Sima and S. Q. Xu, Hematopoiesis toxicity induced by CdTe quantum dots determined in an invertebrate model organism, Biomaterials, 2014, 35, 29422951.

21 E. R. Carmona, A. Creus and R. Marcos, Genotoxic effects of two nickel-compounds in somatic cells of Drosophila melanogaster, Mutat. Res., 2011, 718, 33-37.

22 S. Cherry and N. Silverman, Host-pathogen interactions in drosophila: new tricks from an old friend, Nat. Immunol., 2006, 7, 911-917.

23 P. Irving, J. M. Ubeda, D. Doucet, L. Troxler, M. Lagueux, D. Zachary, J. A. Hoffmann, C. Hetru and M. Meister, New insights into Drosophila larval haemocyte functions through genome-wide analysis, Cell. Microbiol., 2005, 7, 335-350.

24 M. Okada and S. Natori, Purification and characterization of an antibacterial protein from haemolymph of Sarcophaga peregrina (flesh-fly) larvae, Biochem. J., 1983, 211, 727-734.

25 M. Okada and S. Natori, Primary structure of sarcotoxin I, an antibacterial protein induced in the hemolymph of Sarcophaga peregrina (flesh fly) larvae, J. Biol. Chem., 1985, 260, 7174-7177.

26 M. Grigorian and V. Hartenstein, Hematopoiesis and hematopoietic organs in arthropods, Dev. Genes Evol., 2013, 22, 103-115.

27 K. M. Tsoi, Q. Dai, B. A. Alman and W. C. Chan, Are quantum dots toxic? Exploring the discrepancy between cell culture and animal studies, Acc. Chem. Res., 2013, 46, 662-671.

28 Y. Nakahara, Y. Kanamori, M. Kiuchi and M. Kamimura, In vitro studies of hematopoiesis in the silkworm: cell proliferation in and hemocyte discharge from the hematopoietic organ, J. Insect Physiol., 2003, 49, 907-916.

29 Y. Zhong, F. Peng, F. Bao, S. Wang, X. Ji, L. Yang, Y. Su, S. T. Lee and Y. He, Large-scale aqueous synthesis of fluorescent and biocompatible silicon nanoparticles and their use as highly photostable biological probes, J. Am. Chem. Soc., 2013, 135, 8350-8356.

30 Y. He, H. T. Lu, L. M. Sai, W. Y. Lai, Q. L. Fan, L. H. Wang and W. Huang, Synthesis of CdTe nanocrystals through program process of microwave irradiation, J. Phys. Chem. B, 2006, 110, 13352-13356.

31 H. Wang, P. Gao, Y. Wang, J. Guo, K. Q. Zhang, D. Du, X. Dai and G. Zou, Fluorescently tuned nitrogen-doped carbon dots from carbon source with different content of carboxyl groups, APL Mater., 2015, 35, 362.

32 S. Q. Yan, R. Xing, Y. F. Zhou, K. L. Li, Y. Y. Su, J. F. Qiu, Y. H. Zhang, K. Q. Zhang, Y. He, X. P. Lu and S. Q. Xu, Reproductive toxicity and gender differences induced by cadmium telluride quantum dots in an invertebrate model organism, Sci. Rep., 2016, 6, 34182.

33 R. Xing, K. L. Li, Y. F. Zhou, Y. Y. Su, S. Q. Yan, K. L. Zhang, S. C. Wu, Y. H. Sima, K. Q. Zhang, Y. He and S. Q. Xu, Impact of fluorescent silicon nanoparticles on circulating hemolymph and hematopoiesis in an invertebrate model organism, Chemosphere, 2016, 159, 628-637.

34 H. Schefe, K. Lehmann, I. Buschmann, T. Unger and H. Funke-Kaiser, Quantitative real-time RT-PCR data analysis: current concepts and the novel gene expression's CT difference formula, J. Mol. Med., 2006, 84, 901-910.

35 N. Rangarajan, S. Bakshi and J. C. Weisshaar, Localized permeabilization of $\mathrm{E}$. coli membranes by the antimicrobial peptide Cecropin A, Biochemistry, 2013, 52, 6584-6594.

36 Y. Oizumi, H. Hemmi, M. Minami, A. Asaoka and M. Yamakawa, Isolation, gene expression and solution structure of a novel moricin analogue, antibacterial peptide from a lepidopteran insect, Spodoptera litura, Biochim. Biophys. Acta, 2005, 1752, 83-92.

37 K. Ishii, H. Hamamoto, M. Kamimura, Y. Nakamura, H. Noda, K. Imamura, K. Mita and K. Sekimizu, Insect cytokine paralytic peptide (PP) induces cellular and humoral immune responses in the silkworm Bombyx mori, J. Biol. Chem., 2010, 285, 28635-28642.

38 D. H. Kim, R. Feinbaum, G. Alloing, F. E. Emerson, D. A. Garsin, H. Inoue, M. Tanaka-Hino, N. Hisamoto, K. Matsumoto, M. W. Tan and F. M. Ausubel, A conserved p38 MAP kinase pathway in Caenorhabditis elegans innate immunity, Science, 2002, 297, 623-626.

39 D. Liu, Y. Yang, Q. Liu and J. Wang, Inhibition of autophagy by 3-MA potentiates cisplatin-induced apoptosis in esophageal squamous cell carcinoma cells, Med. Oncol., 2011, 28, 105-111.

40 K. Xie, L. Tian, X. Guo, K. Li, J. Li, X. Deng, Q. Li, Q. Xia, Y. Zhong, Z. Huang, J. Liu, S. Li, W. Yang and Y. Cao, BmATG5 and BmATG6 mediate apoptosis following autophagy induced by 20-hydroxyecdysone or starvation, Autophagy, 2016, 12, 381-396.

41 M. M. Ji, J. M. Lee, H. Mo, J. Xu, T. Tatsuke and T. Kusakabe, Proteasome inhibitor MG132 impairs autophagic flux through compromising formation of autophagosomes in Bombyx cells, Biochem. Biophys. Res. Commun., 2016, 479, 690-696.

42 Z. Gai, X. Zhang, M. Islam, X. Wang, A. Li, Y. Yang, Y. Li, J. Peng, H. Hong and K. Liu, Characterization of Atg8 in lepidopteran insect cells, Arch. Insect Biochem. Physiol., 2013, 84, 57-77. 
43 D. Guo and R. Liu, Spectroscopic investigation of the effects of aqueous-phase prepared CdTe quantum dots on protein hemoglobin at the molecular level, J. Biochem. Mol. Toxicol., 2017, DOI: 10.1002/jbt.21953.

44 M. Pohanka, Quantum Dots in the Therapy: Current Trends and Perspectives, Mini-Rev. Med. Chem., 2017, 17, 650-656.

45 M. Hu, J. Yan, Y. He, H. Lu, L. Weng, S. Song, C. Fan and L. Wang, Ultrasensitive, multiplexed detection of cancer biomarkers directly in serum by using a quantum dotbased microfluidic protein chip, ACS Nano, 2010, 4, 488-494.

46 N. L. Teradal and R. Jelinek, Carbon nanomaterials in biological studies and biomedicine, Adv. Healthcare Mater., 2017, DOI: 10.1002/adhm.201700574.

47 P. Xie, Q. Xin, S. T. Yang, T. He, Y. Huang, G. Zeng, M. Ran and X. Tang, Skeleton labeled 13C-carbon nanoparticles for the imaging and quantification in tumor drainage lymph nodes, Int. J. Nanomed., 2017, 12, 4891-4899.

48 M. Kominkova, V. Milosavljevic, P. Vitek, H. Polanska, K. Cihalova, S. Dostalova, V. Hynstova, R. Guran, P. Kopel, L. Richtera, M. Masarik, M. Brtnicky, J. Kynicky, O. Zitka and V. Adam, Comparative study on toxicity of extracellularly biosynthesized and laboratory synthesized CdTe quantum dots, J. Biotechnol., 2017, 241, 193-200.

49 E. Tolstik, L. A. Osminkina, D. Akimov, M. B. Gongalsky, A. A. Kudryavtsev, V. Y. Timoshenko, R. Heintzmann, V. Sivakov and J. Popp, Linear and non-linear optical imaging of cancer cells with silicon nanoparticles, Int. J. Mol. Sci., 2016, 17, E1536, DOI: 10.3390/ijms17091536.

50 J. Liu, F. Erogbogbo, K. T. Yong, L. Ye, J. Liu, R. Hu, H. Chen, Y. Hu, Y. Yang, J. Yang, I. Roy, N. A. Karker, M. T. Swihart and P. N. Prasad, Assessing clinical prospects of silicon quantum dots: studies in mice and monkeys, ACS Nano, 2013, 7, 73037310 .

51 T. Yu, A. Malugin and H. Ghandehari, Impact of silica nanoparticle design on cellular toxicity and hemolytic activity, ACS Nano, 2011, 5, 5717-5728.

52 D. Lison, L. C. Thomassen, V. Rabolli, L. Gonzalez, D. Napierska, J. W. Seo, M. Kirsch-Volders, P. Hoet, C. E. Kirschhock and J. A. Martens, Nominal and effective dosimetry of silica nanoparticles in cytotoxicity assays, Toxicol. Sci., 2008, 104, 155-162.

53 P. Vandenabeele, L. Galluzzi, T. Vanden Berghe and G. Kroemer, Molecular mechanisms of necroptosis: an ordered cellular explosion, Nat. Rev. Mol. Cell Biol., 2010, 11, 700-714.

54 A. Daskalov, B. Habenstein, R. Sabate, M. Berbon, D. Martinez, S. Chaignepain, B. Coulary-Salin, K. Hofmann, A. Loquet and S. J. Saupe, Identification of a novel cell death-inducing domain reveals that fungal amyloid-controlled programmed cell death is related to necroptosis, Proc. Natl. Acad. Sci. U. S. A., 2016, 113, 27202725.
55 T. Dong, D. Liao, X. Liu and X. Lei, Using small molecules to dissect non-apoptotic programmed cell death: necroptosis, ferroptosis, and pyroptosis, ChemBioChem, 2015, 16, 25572561.

56 J. Hitomi, D. E. Christofferson, A. Ng, J. Yao, A. Degterev, R. J. Xavier and J. Yuan, Identification of a molecular signaling network that regulates a cellular necrotic cell death pathway, Cell, 2008, 135, 1311-1323.

57 Y. Zhou, X. Fang, Y. Gong, A. Xiao, Y. Xie, L. Liu and Y. Cao, The Interactions between ZnO Nanoparticles (NPs) and $\alpha$ Linolenic Acid (LNA) Complexed to BSA Did Not Influence the Toxicity of ZnO NPs on HepG2 Cells, Nanomaterials, 2017, 7, 91.

58 H. Yoshida, ER stress and diseases, FEBS J., 2007, 274, 630658.

59 J. Fan, M. Shao, L. Lai, Y. Liu and Z. Xie, Inhibition of autophagy contributes to the toxicity of cadmium telluride quantum dots in Saccharomyces cerevisiae, Int. J. Nanomed., 2016, 11, 3371-3383.

60 S. J. Cho, D. Maysinger, M. Jain, B. Röder, S. Hackbarth and F. M. Winnik, Long-term exposure to CdTe quantum dots causes functional impairments in live cells, Langmuir, 2007, 23, 1974-1980.

61 J. H. Park, L. Gu, G. von Maltzahn, E. Ruoslahti, S. N. Bhatia and M. J. Sailor, Biodegradable luminescent porous silicon nanoparticles for in vivo applications, Nat. Mater., 2009, 8, 331-336.

62 S. Pérez, M. L. Farré and D. Barceló, Analysis, Behavior and ecotoxicity of carbon-based nanomaterials in the aquatic environment, TrAC, Trends Anal. Chem., 2009, 28, 820-832.

63 L. Lai, J. C. Jin, Z. Q. Xu, P. Mei, F. L. Jiang and Y. Liu, Necrotic cell death induced by the protein-mediated intercellular uptake of CdTe quantum dots, Chemosphere, 2015, 135, 240-249.

64 A. M. Derfus, W. C. W. Chan and S. N. Bhatia, Probing the cytotoxicity of semiconductor quantum dots, Nano Lett., 2003, 4, 11-18.

65 R. E. Hancock, A. Nijnik and D. J. Philpott, Modulating immunity as a therapy for bacterial infections, Nat. Rev. Microbiol., 2012, 10, 243-254.

66 M. Feldmann and L. Steinman, Design of effective immunotherapy for human autoimmunity, Nature, 2005, 435, 612-619.

67 K. Huang, H. Ma, J. Liu, S. Huo, A. Kumar, T. Wei, X. Zhang, S. Jin, Y. Gan, P. C. Wang, S. He, X. Zhang and X. J. Liang, Size-dependent localization and penetration of ultrasmall gold nanoparticles in cancer cells, multicellular spheroids, and tumors in vivo, ACS Nano, 2012, 6, 4483-4493.

68 L. Shang, K. Nienhaus and G. U. Nienhaus, Engineered nanoparticles interacting with cells: size matters, $J$. Nanobiotechnol., 2014, 12, 5. 